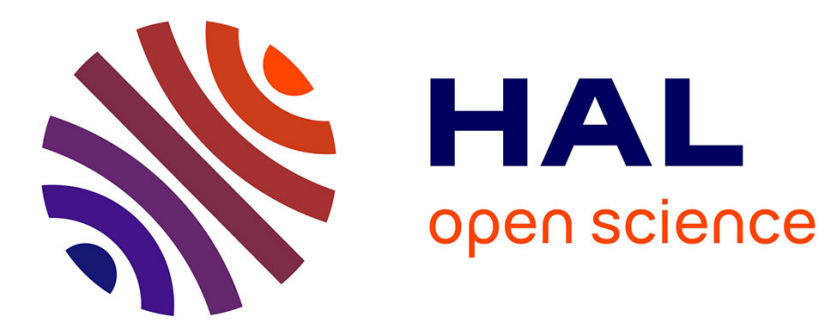

\title{
QUAIL: A Quantitative Security Analyzer for Imperative Code
}

Fabrizio Biondi, Axel Legay, Louis-Marie Traonouez, Andrzej Wasowski

\section{To cite this version:}

Fabrizio Biondi, Axel Legay, Louis-Marie Traonouez, Andrzej Wasowski. QUAIL: A Quantitative Security Analyzer for Imperative Code. CAV 2013 - 25th International Conference on Computer Aided Verification, Jul 2013, Saint Petersburg, Russia. pp.702-707, 10.1007/978-3-642-39799-8_49 . hal-01242615

\section{HAL Id: hal-01242615 https://hal.inria.fr/hal-01242615}

Submitted on 13 Dec 2015

HAL is a multi-disciplinary open access archive for the deposit and dissemination of scientific research documents, whether they are published or not. The documents may come from teaching and research institutions in France or abroad, or from public or private research centers.
L'archive ouverte pluridisciplinaire HAL, est destinée au dépôt et à la diffusion de documents scientifiques de niveau recherche, publiés ou non, émanant des établissements d'enseignement et de recherche français ou étrangers, des laboratoires publics ou privés. 


\title{
QUAIL: A Quantitative Security Analyzer for Imperative Code ${ }^{\star}$
}

\author{
Fabrizio Biondi ${ }^{1}$, Axel Legay ${ }^{2}$, Louis-Marie Traonouez $^{2}$, and Andrzej Wąsowski ${ }^{1}$ \\ 1 IT University of Copenhagen, Denmark \\ 2 INRIA Rennes, France
}

\begin{abstract}
Quantitative security analysis evaluates and compares how effectively a system protects its secret data. We introduce QUAIL, the first tool able to perform an arbitrary-precision quantitative analysis of the security of a system depending on private information. QUAIL builds a Markov Chain model of the system's behavior as observed by an attacker, and computes the correlation between the system's observable output and the behavior depending on the private information, obtaining the expected amount of bits of the secret that the attacker will infer by observing the system. QUAIL is able to evaluate the safety of randomized protocols depending on secret data, allowing to verify a security protocol's effectiveness. We experiment with a few examples and show that QUAIL's security analysis is more accurate and revealing than results of other tools.
\end{abstract}

\section{Introduction}

The Challenge. Qualitative analysis tools can verify the complete security of a protocol, i.e. that an attacker is unable to get any information on a secret by observing the systema property known as non-interference. Non-interference holds when the system's output is independent from the value of the secret, so no information about the latter can be inferred from the former [20]. However, when non-interference does not hold, qualitative analysis cannot rank the security of a system: all unsafe systems are the same.

Quantitative analysis can be used to decide which of two alternative protocols is more secure. It can also asses security of systems that are insecure, but nevertheless useful, in the qualitative sense, such as a password authentication protocol, for which there is always a positive probability that an attacker will randomly guess the password. A quantitative analysis is challenging because it is not sufficient to find a counterexample to a specification to terminate. We need to analyze all possible behaviors of the system and quantify for each one the probability that it will happen and how much of the protocol's secret will be revealed. So far no tool was able to perform this analysis precisely.

Quantitative analysis with QUAIL. We use Quantified Information Flow to reduce the comparison of security of two systems to a computation of expected amount of information, in the information-theoretical sense, that an attacker would learn about the secret by observing a system's behavior. This expected amount of information is known as information leakage $[9,15,8,12,21]$ of a system. It amounts to zero iff the system is

\footnotetext{
* Partially supported by MT-LAB — VKR Centre of Excellence on Modeling of IT
} 
non-interfering [15], else it represents the expected number of bits of the secret that the attacker is able to infer. The analysis generalizes naturally to more than two systems, hence allowing to decide which of them is less of a threat to the secrecy of the data.

To compute information leakage we use a stochastic model of a system as observed by the attacker. The model is obtained by resolving non-determinism in the system code, using the prior probability distribution over the secret values known to the attacker before an attack. Existing techniques represent this with a channel matrix from secret values to outputs [5]. They build a row of the channel matrix for each possible value of the secret, even if the system would behave in the same way for most of them. In contrast, we have proposed an automata based technique [3], using Markovian models. One state of a model represents an interval of values of the secret for which the system behaves in the same way, allowing for a much more compact and tractable representation.

We build a Markov chain representing the behavior observed by the attacker, then we hide the states that are not observable by the attacker, obtaining a smaller Markov chain-an observable reduction. Then we calculate the correlation between the output the attacker can observe and the behavior dependent on the secret, as it corresponds to the leakage. Since leakage in this case is mutual information, it can be computed by adding the entropy of the observable and secret-dependent views of the system and subtracting the entropy of the behavior depending on both. See [3] for details.

QUAIL (QUantitative Analyzer for Imperative Languages) implements this method. It is the first tool supporting arbitrary-precision quantitative evaluation of information leakage for randomized systems or protocols with secret data, including anonymity and authentication protocols. Systems are specified in a simple imperative modeling language further described on QUAILS website.

QUAIL performs a white-box leakage analysis assuming that the attacker has knowledge of the system's code but no knowledge of the secret's value, and outputs the result and eventually infor-

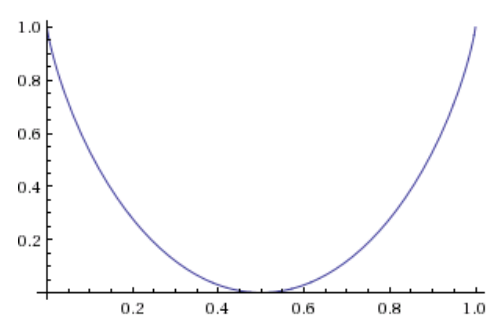

Fig. 1: Bit XOR leakage as a function of $\operatorname{Pr}(r=1)$ mation about the computation, including the Markov chains computed during the process.

Example. Consider a simple XOR operation example. Variable $h$ stores a 1-bit secret. The protocol generates a random bit $r$, where $r=1$ with probability $p$. It outputs the result of exclusive-or between values of $h$ and $r$. The attacker knows $p$ and can observe the output, so if $h=r$, but not the values of $r$ or $h$.

If $p=0.5$ the attacker cannot infer any information about $h$, the leakage is zero bits (non-interference). If $p=0$ or $p=1$ then she can determine precisely the value of $h$, and thus the leakage is 1 bit. This can be verified efficiently with language-based tools like APEX [10]. However, QUAIL is the only tool able to precisely compute the leakage for all possible values of $p$ with arbitrary precision. Figure 1 shows that XOR protocol leaks more information as the value of $r$ becomes more deterministic. For instance $p=0.4$ is safer than $p=0.8$. 


\section{QUAIL Implementation}

The input model is specifed in QUAIL's imperative language designed to facilitate succinct and direct modeling of protocols, providing features such as arbitrary-size integer variables and arrays, random assignments, while and for loops, named constants and conditional statements. Figure 2 presents the input code for the bit XOR example.

For a given input code QUAIL builds an annotated Markov chain representing all possible executions of the protocol, then modifies it to encode the protocol when observed by the attacker whose aim is to discover the protocol's secret data. Finally, QUAIL extracts a model of the observable and secret-dependent behavior of the system, and computes the correlation between them, which is equivalent to the amount of bits of the secret that the attacker can infer by observing the system. We now discuss QUAIL implementation following the five steps of the method proposed in [3]:

Step 1: Preprocessing. QUAIL translates the input code into a simplified internal language. It rewrites conditional statements and loops (if, for and while) to conditional jumps (if-goto) and substitutes values for named constant references.

Step 2: Probabilistic symbolic execution. QUAIL performs a symbolic forward execution of the input program constructing its semantics as a finite Markov chain (a fully probabilistic transition system) with a single starting state. To this end, QUAIL needs to know the attacker's probability distributions over the secret variables. For each conditional branch, we compute the conditional probability of the guard being satisfied given the values of the public variables and the probability distributions over the secret variables. Then QUAIL generates two successor states, one for the case in which the guard is satisfied and one when not satisfied. This is the most time-consuming step, so QUAIL uses an on-the-fly optimization to avoid building internal states that would be removed in the next step. For instance, it does not generate new states for assignments to a non-observable public variable. Instead it changes the value of the variable in the current state.

Step 3: State hiding and model reduction. To represent what the attacker can examine, QUAIL reduces the Markov chain model by iteratively hiding all unobservable states. For the standard attacker, these are all the internal states, i.e. all the states except the initial and the output states. A state is hidden by creating transitions from its predecessors to its successors and removing it from the model. This operation normally eliminates more than $90 \%$ of the states of the Markov chain model, building its observable reduction. This operation also detects non-terminating loops and collapses then in a single nontermination state. States are equipped with a list of their predecessors and successors to quicken this step. An observable reduction looks like a probability distribution from the starting states to the output states, since all other states are hidden.

Step 4: Quotienting. Recall from Sect.1 that we have to quantify the correlation between the observable and secret-dependent views of the system. QUAIL relies on the notion of quotients to represent different views of the system and compute their correlation. A quotient is a Markov chain obtained by merging together states in the observable 
Table 1: QUAIL analysis of the leakage in an authentication program

\begin{tabular}{lcccc}
\hline Password length & 2 & 32 & 64 & 500 \\
\hline Leakage & $8.11 \cdot 10^{-1}$ & $7.78 \cdot 10^{-9}$ & $3.54 \cdot 10^{-18}$ & $1.52 \cdot 10^{-148}$ \\
\hline
\end{tabular}

inherent leakage, such as authentication protocols. For instance, with a simple password authentication, the user inputs a password and is granted access privilege if the password corresponds to the secret stored in the system. The chance of an attacker guessing a password is always positive (although it depends on the password's length). Also, even if the attacker gets rejected she learns something about the secret- the fact that the attempted value was not correct. QUAIL can quantify the precise leakage as a function of the bit length of the password, as shown in Table 1.

Existing qualitative tools can establish whether a protocol is completely secure or not, i.e. whether it respects non-interference. They cannot discriminate protocols that allow acceptable and unacceptable violations of non-interference. APEX [10] is an analyzer for probabilistic programs that can check programs equivalence, while PRISM [14] is a probabilistic model-checker. With these tools authentication protocols will always be flagged as unsafe, and a comparison between them is impossible.

QUAIL can be used also to analyze anonymity protocols, like the grade protocol and the dining cryptographers [6]. The interested reader can find discussion and input code for these examples on the QUAIL website. These protocols provide full anonymity on the condition that some random data is generated with a uniform probability distribution; their effectiveness in these cases can be efficiently verified with the qualitative tools above. If the probability distribution over the random data is not uniform some private data is leaked, and QUAIL is again the only tool that can quantify this leakage. Presently, the models for these protocols tend to grow exponentially, so the analysis becomes time-consuming already for about 6-7 agents.

Qualitative tools and technique are more closely related to QUAIL; we present some of them and discuss the main differences. It is worth noting that most of them either do not work for analyzing probabilistic programs $[1,11,13,17]$ or are based on a channel matrix with an impractical number of lines [4,7].

JPF-QIF [19] is a tool that computes an upper bound of the leakage by estimating the number of possible outputs of the system. JPG-QIF is much less precise than QUAIL, and it is not able for instance to prove that the security of an authentication increases by increasing the password size.

McCamant and Ernst [16] and Newsome, McCamant and Song [18] propose quantitative extensions of taint analysis. This approach, while feasible even for large programs, still does not allow to analyze probabilistic programs, making it unsuitable for security protocols.

Bérard et al. propose a quantification of information leakage based on mutual information, though they name it restrictive probabilistic opacity [2] and do not refer to some of the core papers of the subject, like the works of Clark, Hunt and Malacaria [8,9]. The approach tries to quantify leakage on probabilistic models, and is thus phylosophically close to ours. They compute mutual information as the expected difference between 
prior and posterior entropy, and since the latter depends on all possible values of the secret we expect that an eventual implementation would be in general very inefficient compared to the QUAIL quotient-based approach.

\section{References}

1. Michael Backes, Boris Köpf, and Andrey Rybalchenko. Automatic discovery and quantification of information leaks. In IEEE Symposium on Security and Privacy, 2009.

2. Béatrice Bérard, John Mullins, and Mathieu Sassolas. Quantifying opacity. In G. Ciardo and R. Segala, editors, QEST'10. IEEE Computer Society, September 2010.

3. Fabrizio Biondi, Axel Legay, Pasquale Malacaria, and Andrzej Wasowski. Quantifying information leakage of randomized protocols. In Roberto Giacobazzi, Josh Berdine, and Isabella Mastroeni, editors, VMCAI. Springer, 2013.

4. Konstantinos Chatzikokolakis, Tom Chothia, and Apratim Guha. Statistical measurement of information leakage. In Javier Esparza and Rupak Majumdar, editors, TACAS, 2010.

5. Konstantinos Chatzikokolakis, Catuscia Palamidessi, and Prakash Panangaden. Anonymity protocols as noisy channels. In Information and Computation. Springer, 2006.

6. David Chaum. The dining cryptographers problem: Unconditional sender and recipient untraceability. Journal of Cryptology, 1:65-75, 1988

7. Tom Chothia and Apratim Guha. A statistical test for information leaks using continuous mutual information. In CSF, pages 177-190. IEEE Computer Society, 2011.

8. David Clark, Sebastian Hunt, and Pasquale Malacaria. Quantitative analysis of the leakage of confidential data. Electr. Notes Theor. Comput. Sci., 59(3):238-251, 2001.

9. David Clark, Sebastian Hunt, and Pasquale Malacaria. A static analysis for quantifying information flow in a simple imperative language. Journal of Computer Security, 15, 2007.

10. S. Kiefer, A. S. Murawski, J. Ouaknine, B. Wachter, and J. Worrell. Apex: An analyzer for open probabilistic programs. In Proc. CAV'12, LNCS. Springer, 2012.

11. Vladimir Klebanov. Precise quantitative information flow analysis using symbolic model counting. In Fabio Martinelli and Flemming Nielson, editors, QASA, 2012.

12. Boris Köpf and David A. Basin. An information-theoretic model for adaptive side-channel attacks. In ACM Conference on Computer and Communications Security, 2007.

13. Boris Köpf, Laurent Mauborgne, and Martín Ochoa. Automatic quantification of cache side-channels. In P. Madhusudan and Sanjit A. Seshia, editors, CAV, 2012.

14. M. Kwiatkowska, G. Norman, and D. Parker. PRISM 4.0: Verification of probabilistic real-time systems. In Proc. CAV'11, volume 6806 of LNCS. Springer, 2011.

15. Pasquale Malacaria. Algebraic foundations for information theoretical, probabilistic and guessability measures of information flow. CoRR, abs/1101.3453, 2011.

16. Stephen McCamant and Michael D. Ernst. Quantitative information flow as network flow capacity. In Rajiv Gupta and Saman P. Amarasinghe, editors, PLDI. ACM, 2008.

17. Chunyan $\mathrm{Mu}$ and David Clark. A tool: Quantitative analyser for programs. In QEST. IEEE Computer Society, 2011.

18. James Newsome, Stephen McCamant, and Dawn Song. Measuring channel capacity to distinguish undue influence. In S. Chong and D. A. Naumann, editors, PLAS. ACM, 2009.

19. Quoc-Sang Phan, Pasquale Malacaria, Oksana Tkachuk, and Corina S. Pasareanu. Symbolic quantitative information flow. ACM SIGSOFT Software Engineering Notes, 37(6):1-5, 2012.

20. Andrei Sabelfeld and Andrew C. Myers. Language-based information-flow security. IEEE Journal on Selected Areas in Communications, 21(1):5-19, 2003.

21. Geoffrey Smith. On the foundations of quantitative information flow. In Luca de Alfaro, editor, FOSSACS, volume 5504 of LNCS, pages 288-302. Springer, 2009. 


\section{A Running QUAIL through an example}

We detail in this appendix the result of the computations performed by QUAIL when analyzing the bit XOR example presented in Fig. 2. We consider the value of 0.8 for the random bit probability on line 4. We launch QUAIL as explained in Section 3:

quail bit_xor.quail -p $3-\mathrm{v} \quad 0-\mathrm{mc} \quad 0 \quad 1 \quad 2 \quad 3 \quad 4$-Tpdf

This will compute the leakage of the program with a precision of 3 digits for the

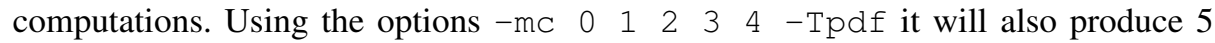
Markov chains in a PDF format, corresponding to the different intermediate steps of the computation. As a result the tool outputs the leakage of the program which is:

0.28

To compute this result Step 1 converts the input program given on the left of Fig. 2 into a preprocessed program shown on the right. This program is written in a separate file bit_xor.quail.pp.

In Step 2, QUAIL parses the preprocessed code and builds the Markov chain model shown in Fig. 3 that corresponds to all the executions of the program. Note that public variables are labeled with a precise value in each state (e.g. $l=0)$ while private variables have intervals of allowed values (e.g. $h=[0,1])$.

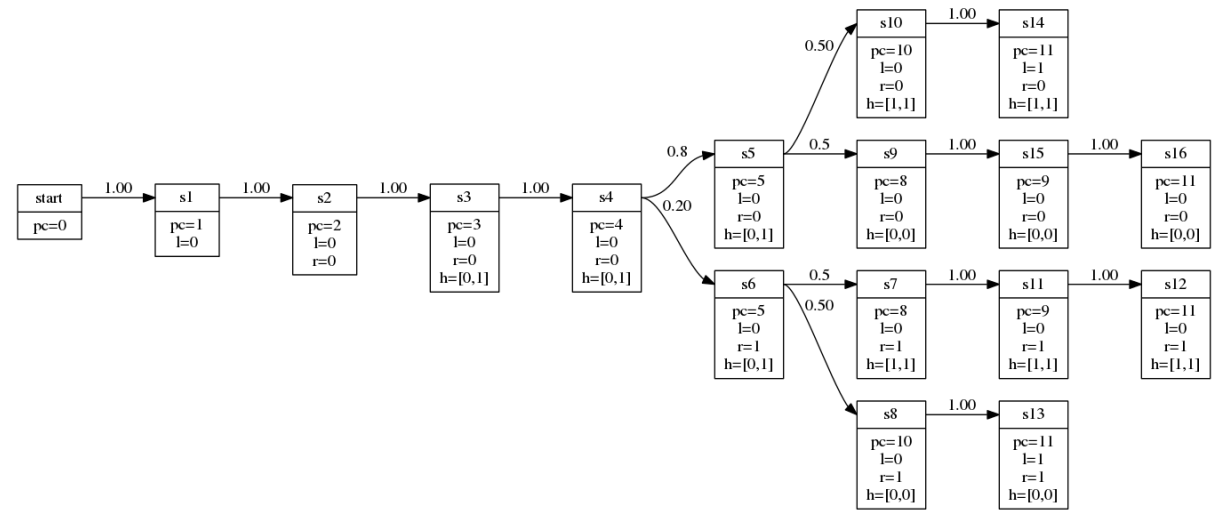

Fig. 3: Bit XOR example: Markov chain model of the program

In Step 3, QUAIL hides all non observable states, i.e. the internal states, and produces a Markov chain with only transitions between the initial states and the output states, as shown in Fig. 4.

In Step 4, QUAIL computes the quotient Markov chains. In the attacker's quotient, since in both states s 13 and s 14 the observable variable $l$ equals to 1 , these two states are merged together, and similarly for the states s 12 and s 16 in which $l=0$. The result is shown in Fig. 5a.

In the secret's quotient, the states $\mathrm{s} 12$ and $\mathrm{s} 14$ share the value $h=0$ for the secret variable, whereas the states s13 and s 16 share the value $h=1$. These states are merged together as shown in Fig. 5b. 


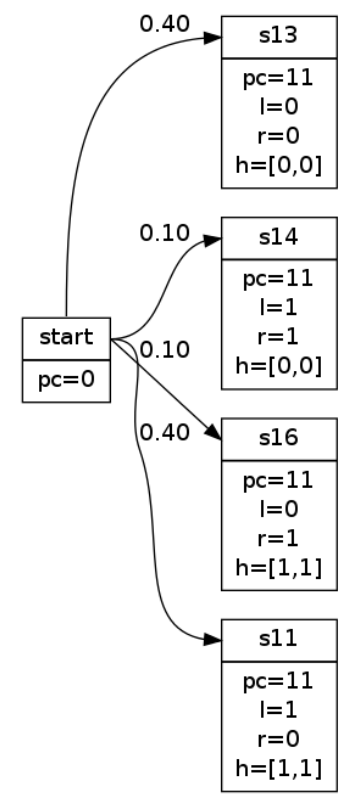

Fig. 4: Bit XOR example: observable reduction of the Markov chain model

Finally, in the joint quotient, we consider the intersection of the two previous discrimination relations, i.e. only states with the same value to both observable and secret variables can be merged together. This is not the case for any state in the observable reduction, and therefore the joint quotient Markov chain shown in Fig. $5 \mathrm{c}$ is similar to the original observable reduction of Fig. 4.

In the final Step 5, QUAIL computes the entropy of the three quotients Markov chains. The entropy of the attacker's quotient is:

$$
H_{a}=-0.5 * \log (1 / 0.5)-0.5 * \log (1 / 0.5)=1
$$

The entropy of the secret's quotient is the same:

$$
H_{s}=-0.5 * \log (1 / 0.5)-0.5 * \log (1 / 0.5)=1
$$

The entropy of the joint quotient is:

$$
H_{j}=-0.4 * \log (1 / 0.4) * 2-0.1 * \log (1 / 0.1) * 2=1.058+0.664=1.722
$$

The information leakage is the sum of the first two entropies minus the entropy of the joint quotient, therefore:

$$
L=H_{a}+H_{s}-H_{j}=2-1.722=0.278
$$

This result is rounded to 0.28 in the final output. 


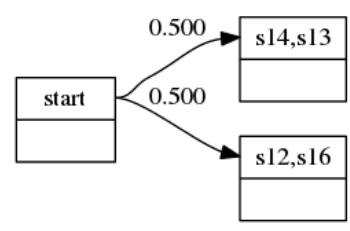

(a) Attacker's quotient

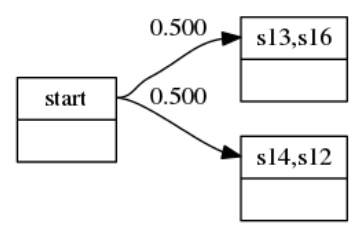

(b) Secret's quotient

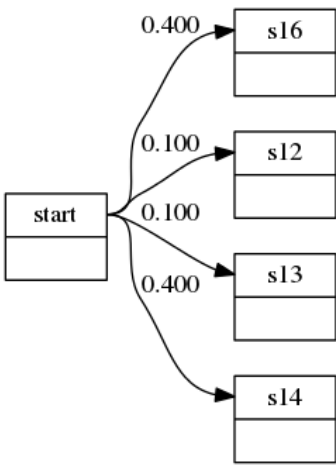

(c) Joint quotient

Fig. 5: Markov chains of the quotients

\section{B QUAIL imperative language}

\section{B.1 Variable declarations}

All variables in QUAIL are fixed sized integers. They are declared at the beginning of the program. Constants can be declared in the following manner:

const $\mathrm{N}:=4$;

They are replaced by their value during the preprocessing step.

Public variables are either public or observable. In the latter case the attacker will be able to distinguish their value. They are declared in the following manner:

public int 4 var; or observable int 4 var;

declares a 4 bits integer variable whose name is var, either public or observable.

public int 4 var := 5;

declares var and initializes it to value 5. Any expression can be used to initialized a variable, provided that the variables used in the expression are public or constants and have been previously declared. Variables not initialized are implicitly initialized to the value 0 .

Private variables are either private or secret. The attacker will only infer knowledge on the latter. They are declared in the following manner:

private int 4 var; or secret int 4 var;

declares a 4 bits integer variable whose name is var, either private or secret.

private int 4 var $:=[0,1][2,5]$;

declares var and restricts its range to the two intervals [0,1] and [2,5]. Again any expression can be used in the bounds of the intervals.

\section{B.2 Arrays}

Variables can also be arrays of integers and multi-dimensional arrays. Arrays are declared in addition to the integer type of a variable. 
public array[4] of int4 tab;

declares a public variable tab that is an array of 4 bits integer of size 4 whose indices range from 0 to 3 , while

public array[1..4] of int4 tab;

declares tab as an array of size 4 whose indices range from 1 to 4 . The size of an array can be any expression that evaluates to an integer.

Arrays are replaced during the preprocessing. Therefore, an array variable named tab, whose indices range from 0 to 3 , declares 4 variables, whose name are tab [0], tab [1], tab [2] and tab [3]. They have the same publicity and the same integer type as the array.

An array may be initialized with a set of initial values:

public array [1..4] of int 4 tab := $\{1,1,2,2\}$;

initializes tab such that tab [1] and tab [2] are equal to 1, while tab [3] and tab [ 4 ] are equal to 2 . Private arrays can be initialized like any private variable, with a set of intervals:

private array [1..4] of int 4 tab := $[0,1]$;

In that case all the variables in the array are initialized to the same range of integers.

\section{B.3 Expressions}

Expressions are used in guards, assignments, variables initialization and arrays indices. Binary operators $(||, \& \&, \wedge,+,-, *, /$ and $\circ)$ and unary operators $(-, !)$ can used. Classical operators precedence is assumed. For boolean operations integer variables are considered as a true value if non null, and false if null. Only public variables, constants and integers can be used in expressions.

\section{B.4 Guards}

Guards are limited to a single comparison between a variable on the left side (either public, or private, or constant, or an integer value) and an expression on the right side. Any comparison operator among $<,>,<=,>=,==$ and $!=$ can be used.

\section{B.5 Assignments}

An assignment statement is written in the following manner: assign var := expr;

where var is a public variable (possibly with indices) and expr is an expression containing no private variables.

\section{B.6 Random assignments}

The program can used two types of random primitives to assign values to a variable. random var := random (expr_min, expr_max); assigns to a public variable var a random value, chosen between the values of expr_min and expr_max, with a uniform probability distribution. 
random var := randombit $(\mathrm{p})$;

where $\mathrm{p}$ is a float value lower than 1 , assigns to a public variable var a random bit value, that is 0 with probability $\mathrm{p}$, and 1 with probability $1-\mathrm{p}$.

\section{B.7 IF statements}

IF conditional statements starts with the keyword if, possibly followed by elif and else, and ends with $f i$. The consequent statements are listed after the keyword then. For example the following structures are allowed:

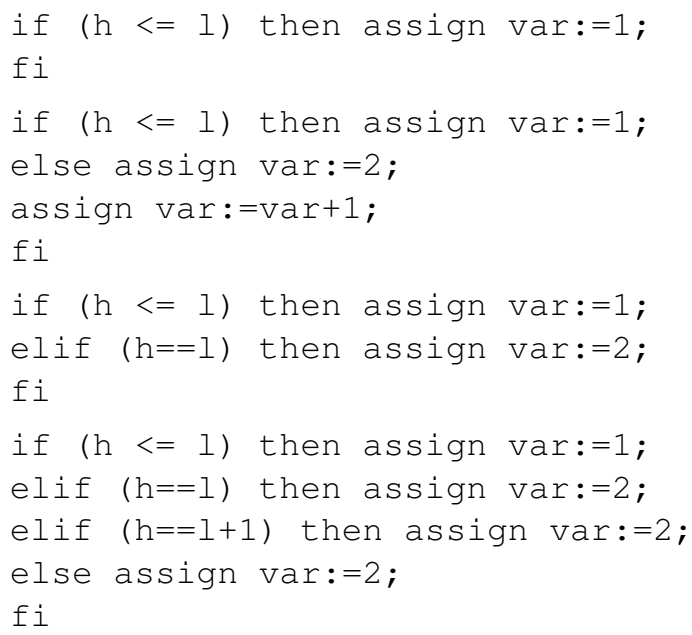

\section{B.8 WHILE statements}

Conditional WHILE loop starts with the keyword while, followed by a guard, and the statements included in the loop are listed between the keywords do and od. For example the following structure is allowed:

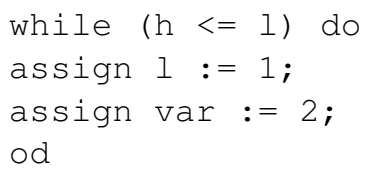

\section{B.9 FOR statements}

A FOR loop can be used to browse all the elements of an array. The syntax is:

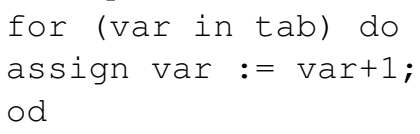

The variable var is a local variable that must only be used inside the loop. It will take successively each value in the array tab. Note that if tab is a multi-dimensional array var is also an array. 


\section{B.10 Return statements}

The program ends when a return statement is reached. Its syntax is simply: return;

\section{Further examples}

\section{C.1 The Grade protocol}

In the Grades protocol a group of $k$ students $s_{1}, \ldots, s_{k}$ is given each a secret grade $g_{i}$ between 0 and $m-1$. The students want to compute the sum of their grades without disclosing them. To this aim they produce $k$ random numbers between 0 and $n=$ $(m-1) * k+1$ such that the number $r_{i}$ is known only to the students $s_{i}$ and $s_{(i+1) \% k}$. Then each student $s_{i}$ outputs a number $d_{i}=g_{i}+r_{i}-r_{(i+1) \% k}$, and the sum of all grades is equivalent to the sum of the outputs modulo $n$. The input code for the Grade protocol is shown on Fig. 6 on the left.

To prove the security of the protocol, and thus the secrecy of the grades, we need to show that the information the attacker gains by knowing the declarations and the sum is the same as the information he would gain by knowing only the sum; the input code for the latter system is shown in Fig. 6 on the right. The leakage of the protocol for different numbers of students and grades is shown in Table 2(a); the leakage of the protocol declaring only the final sum is shown in Table 2(b). The tables are identical, demonstrating that when the attacker knows the students' declarations and the sum of the grades, she does not learn more information than the sum of the grades.

Table 2: Grades: leakage tables for attacker knowing a) the outputs and b) the sum only

(a)

\begin{tabular}{cccc}
\hline \multicolumn{4}{c}{ Students } \\
\hline $\mathbf{2}$ & $\mathbf{3}$ & $\mathbf{4}$ & $\mathbf{5}$ \\
\hline
\end{tabular}

21.5001 .8112 .0302 .198

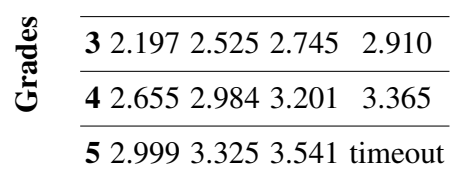

(b)

\begin{tabular}{cccc}
\hline \multicolumn{4}{c}{ Students } \\
\hline $\mathbf{2}$ & $\mathbf{3}$ & $\mathbf{4}$ & $\mathbf{5}$
\end{tabular}

21.5001 .8112 .0302 .198

\begin{tabular}{|c|c|}
\hline 32.1972 .5252 .745 & 2.910 \\
\hline 42.6552 .9843 .201 & 3.365 \\
\hline
\end{tabular}

\section{C.2 The Dining Cryptographers protocol}

The Dining Cryptographers protocol is an anonymity protocol in which a number of agents collaborate to a shared computation depending on each agent's secret [6]. A group of $n$ cryptographers is dining around a round table. At the end of the dinner, the waiter informs them that the bill has already been settled by someone who would prefer to remain anonymous. The cryptographers respect the payer's wish for anonymity, 
Some results are shown in Fig. 7 on the right; for different numbers of cryptographers we show that as the probability of the coin toss approaches 0 or 1 the leakage increases. When it is 0 or 1 the leakage is equivalent to the bit size of the secret, i.e. the logarithm in base 2 of $n+1$, proving that the whole secret gets leaked, and thus the attacker learns the identity of the payer, whoever he is.

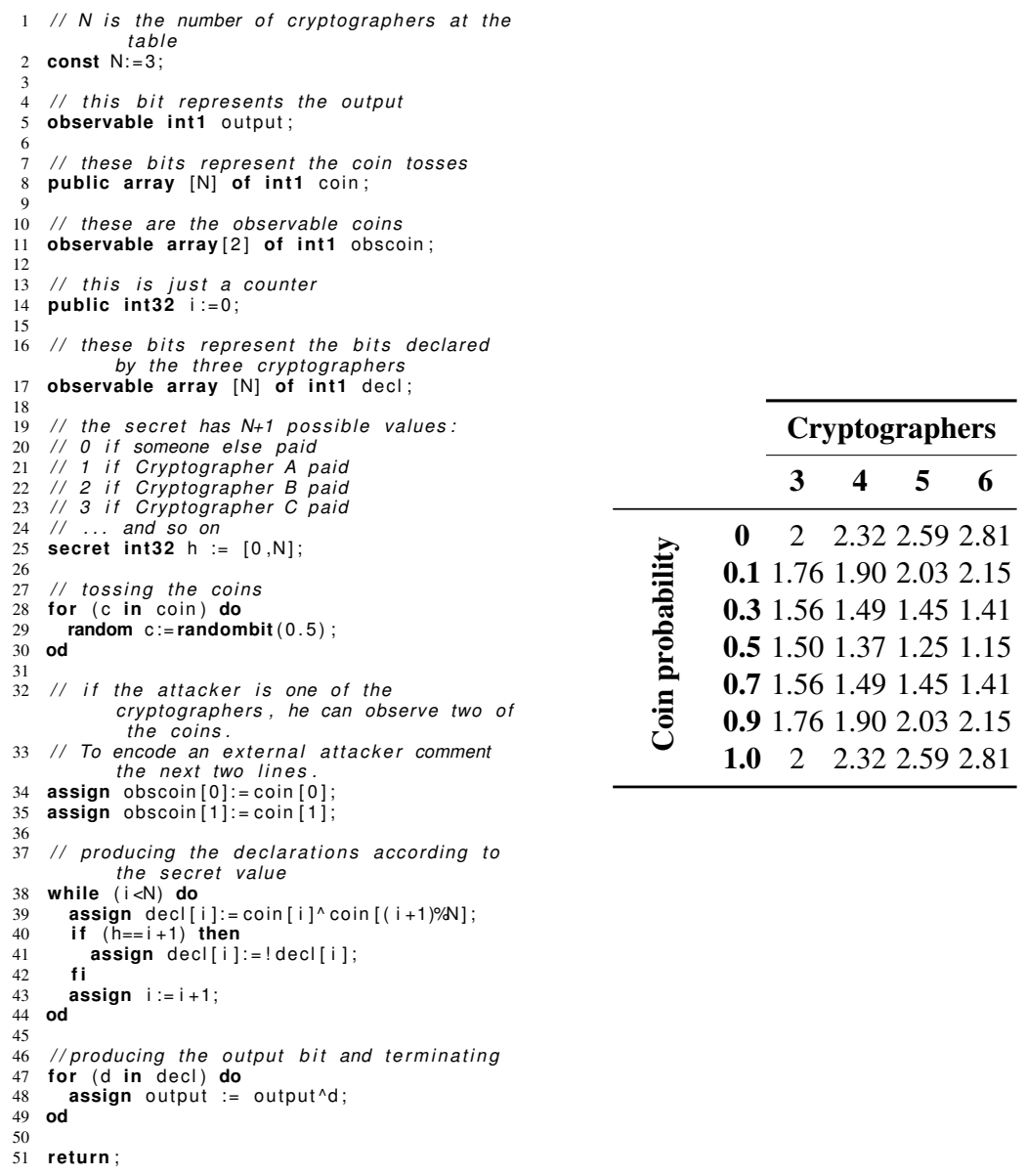

Fig. 7: Dining Cryptographers example: input code for the Dining Cryptographers (on the left) and leakage table as a function of the number of cryptographers and of the probability of the random coin toss (on the right). 